\title{
Association Between Autistic Symptoms and Self-Stigma in Patients with Schizophrenia Spectrum Disorders
}

This article was published in the following Dove Press journal: Neuropsychiatric Disease and Treatment

\author{
Hiroshi Komatsu, \\ Takashi Ono, 2 \\ Yoshinori Maita, ${ }^{3}$ \\ Yusuke Ishida, ${ }^{4}$ \\ Tatsuo Kikuchi, ${ }^{2}$ \\ Takahiro Maki, ${ }^{5}$ Satoshi Hase, ${ }^{6}$ \\ Hisakazu Sakurai, ${ }^{6}$ \\ Akiko Oba, ${ }^{5}$ \\ Osamu Teshirogi, ${ }^{6}$ \\ Akira Suzuki, ${ }^{3}$ Yasuko Mori, ${ }^{3}$ \\ Chikako Shoji, ${ }^{3}$ Akira Fujita, ${ }^{3}$ \\ Sachiko Takahashi, ${ }^{3}$ \\ Takayuki Ebina, ${ }^{3}$ Shinya Ozaki, ${ }^{3}$ \\ Ryuta Honma, ${ }^{3}$ \\ Hiroaki Tomita, ${ }^{7}$ \\ Yoshihisa Kakuto ${ }^{2}$ \\ 'Department of Psychiatry, Tohoku \\ University Hospital, Sendai, Miyagi, Japan; \\ ${ }^{2}$ Department of Psychiatry, Miyagi \\ Psychiatric Center, Natori, Miyagi, Japan; \\ ${ }^{3}$ Department of Nursing, Miyagi Psychiatric \\ Center, Natori, Miyagi, Japan; ${ }^{4}$ Department \\ of Pharmacy, Miyagi Psychiatric Center, \\ Natori, Miyagi, Japan; ${ }^{5}$ Department of \\ Rehabilitation, Miyagi Psychiatric Center, \\ Natori, Miyagi, Japan; ${ }^{6}$ Department of \\ Social Life Support, Miyagi Psychiatric \\ Center, Natori, Miyagi, Japan; ${ }^{7}$ Department \\ of Psychiatry, Graduate School of Medicine, \\ Tohoku University, Sendai, Miyagi, Japan
}

Correspondence: Hiroshi Komatsu Department of Psychiatry,

Tohoku University Hospital, Sendai,

Miyagi 980-8574, Japan

Tel +8I-22-717-7262

Fax +8I-22-7I 7-7266

Email hkomatsul019@gmail.com
Purpose: Self-stigma negatively influences self-esteem, quality of life, self-efficacy, treatment adherence, and recovery in psychiatric patients. By revealing personality traits that influence self-stigma, we can gain useful knowledge for the management of self-stigma. A previous meta-analysis indicated that patients with schizophrenia have higher scores on the Autism-Spectrum Quotient (AQ) than healthy controls. However, the relationship between autistic symptoms and self-stigma in patients with schizophrenia spectrum disorders remains unclear. Therefore, the present study aimed to reveal the association between autistic symptoms and self-stigma in patients with schizophrenia spectrum disorders.

Patients and Methods: We recruited 127 patients with schizophrenia spectrum disorders (schizophrenia, schizoaffective disorder, and delusional disorder). We assessed participants' self-stigma and autistic symptoms using the Internalized Stigma for Mental Illness (ISMI) scale and the Autism-Spectrum Quotient (AQ), respectively. The differences in the scores of ISMI and AQ according to patient characteristics were investigated. Multiple regression analysis controlling for age and gender was performed to determine the relationship between the total scores on the AQ and IMSI scale.

Results: Female patients showed a higher level of self-stigma than males. Unmarried patients showed a significantly higher score on the AQ than married patients. Multiple regression analysis adjusted for age and gender indicated that the total score on AQ might be a predictor of the overall rating on ISMI in patients with schizophrenia spectrum disorders.

Conclusion: This study is the first to reveal the association between autistic symptoms and self-stigma in patients with schizophrenia spectrum disorders. Our results highlight the importance of considering autistic symptoms in the assessment and management of selfstigma in patients with schizophrenia spectrum disorders.

Keywords: schizophrenia spectrum disorders, self-stigma, autistic symptoms, gender difference, marital status

\section{Introduction}

Research has found that stigma associated with patients with mental illnesses is a global problem. ${ }^{1}$ Patients with serious mental illnesses, such as schizophrenia, have reportedly faced different forms of stigmatization. ${ }^{2,3}$ Corrigan et al categorized stigma types into "public", "self-stigma" and "label avoidance. “4 Self-stigma develops when people with mental illnesses apply the stigma projected by the general population has toward them, to themselves. ${ }^{5}$ Watson et al hypothesized that self-stigma occurs through 
three processes: 1) stereotype awareness, 2) stereotype agreement, and 3) self-concurrence. ${ }^{6}$ Self-stigma is associated with several detrimental consequences (eg, diminished selfesteem, lower quality of life (QOL) and self-efficacy, and more depressive symptoms). ${ }^{6-12}$ Moreover, self-stigma can also be a barrier to recovery and treatment adherence. ${ }^{13-17}$ However, not all patients diagnosed with schizophrenia internalize stigma. ${ }^{18}$ Prior studies have reported an association between personality traits (ie, harm-avoidance and selfdirectedness) and self-stigma. ${ }^{10,19-21}$ These results suggest the importance of considering personality traits in the assessment and management of self-stigma in people with mental illnesses.

Murai et al found that patients with schizophrenia have higher scores on the Autism-Spectrum Quotient (AQ) than healthy people, and lower AQ scores than people with autism. $^{22}$ A recent meta-analysis also revealed higher levels of autistic symptoms in patients with schizophrenia spectrum disorders than in healthy control subjects. ${ }^{23}$ In the metaanalysis, four subscales of AQ (social skills, attention switching, communication skills, and imagination) showed significant differences between the scores of patients with schizophrenia spectrum disorders and healthy individuals.

In spite of previous studies on related topics, the relationship between autistic symptoms and self-stigma in patients with schizophrenia spectrum disorders remains unclear. Therefore, the present study aimed to reveal the association between autistic symptoms and self-stigma in patients with schizophrenia spectrum disorders. We hypothesized that schizophrenia spectrum disorder patients with a higher level of autistic symptoms will have difficulty coping with stigmatization due to lower communication skills and reduced cognitive flexibility, and are more likely to internalize stigma than those with fewer autistic symptoms.

\section{Patients and Methods}

\section{Patients}

We recruited 136 patients with schizophrenia spectrum disorders from January 2018 to November 2019 at the Miyagi Psychiatric Center. Psychological assessment data were collected from each patient. Data were successfully obtained from 127 patients ( 5 patients withdrew consent and 4 patients did not complete the questionnaires). Participants were diagnosed with schizophrenia, schizoaffective disorder, or delusional disorder by two psychiatrists using the Diagnostic and Statistical Manual of Mental Disorders Fifth Edition (DSM-
5). Patients with intellectual disabilities, organic mental disorders, or younger than 20 years old, were excluded.

After the study procedures were explained, written informed consent was obtained from all participants in accordance with the Declaration of Helsinki (1991). This study was approved by the Ethics Committee of Miyagi Psychiatric Center (MPC-20,171,124, 20,190,320)

\section{Psychological Assessment}

We assessed self-stigma using the Internalized Stigma for Mental Illness (ISMI) scale developed by Ritsher et al. ${ }^{11}$ The ISMI scale is a four-point Likert scale ranging from 1 (strongly disagree) to 4 (strongly agree). It consists of 29 items on five subscales: alienation (6 items), stereotype endorsement ( 7 items), perceived discrimination (5 items), social withdrawal (6 items), and stigma resistance (5 items). The items of the stigma resistance subscale are scored in reverse. The higher the total score on the ISMI scale, the more severe is the internalized stigma. Tanabe et al tested the reliability and validity of the Japanese version of the ISMI scale (ISMI-J). ${ }^{24}$ The internal consistency (Cronbach's $\alpha$ ) for ISMI-J was 0.913 in the present study.

We evaluated autistic symptoms in patients using the Autism-Spectrum Quotient (AQ) developed by BaronCohen and colleagues. ${ }^{25}$ The AQ is a 50 -item self-report scale divided into five subscales: social skills, attention switching, attention to detail, communication skills, and imagination. Each item is rated on a four-point scale with the following response options: "definitely agree," "slightly agree," "slightly disagree," and "definitely disagree." "Definitely agree" and "slightly agree" are scored as "1", and "slightly disagree," and "definitely disagree" are scored as " 0 " in 24 items (No. 2, 4-7, 9, 12, 13, 16, 18 $-23,26,33,35,39,41-43,45$, and 46). The remaining 26 items are reversely scored. Wakabayashi et al tested the reliability and validity of the Japanese version of the AQ (AQ-J). ${ }^{26}$ In this study, the Cronbach's $\alpha$ for AQ-J was 0.714 .

\section{Demographic Variables}

We investigated age, gender, age of onset, current duration of hospitalization period, number of hospitalizations, years of education, marital status, and partners as patient demographics.

\section{Statistical Analysis}

Statistical evaluations were performed using SPSS Statistics 24 (Japan IBM, Tokyo, Japan). 
We categorized participants into three groups (outpatients, short-stay inpatients, and long-stay inpatients). Demographic variables among the groups were compared using Fisher's exact test, analysis of variance (ANOVA), Kruskal-Wallis test, Mann-Whitney U-test, or unpaired $t$-test, as appropriate. We compared the ISMI-J scores and the AQ-J scores based on the demographic variables using the Kruskal-Wallis test, Mann-Whitney U-test, or unpaired $t$-test, appropriately.

We performed Spearman's bivariate correlation analysis to examine the correlations between the ISMI-J scores, clinical variables, and the AQ-J scores. Multiple regression analyses were performed with age and gender as covariates to investigate the association between the total score on the ISMI scale and the overall AQ score. Bonferroni corrections were applied for post hoc multiple comparison tests. Statistical significance was defined as a two-tailed p-value $<0.05$.

\section{Results}

\section{Patient Characteristics}

Data was successfully obtained from 127 participants with schizophrenia spectrum disorders (124 were diagnosed with schizophrenia, 3 with schizoaffective disorder, and 1 with delusional disorder). The mean age ( \pm standard deviation, SD) was $46.5 \pm 13.8$ years. There were no significant differences in gender, age, diagnosis, years of education, and partners among outpatients, short-stay inpatients (patients hospitalized for less than six months [in days: mean $\pm \mathrm{SD}=53 \pm 66.0]$ ), and long-stay inpatients (patients hospitalized for more than one year [in days: mean $\pm \mathrm{SD}=$ 1931.4 \pm 2609.8]) (Table 1). In contrast, there was a significant difference observed in the age of onset among the three groups (Kruskal-Wallis test $[\mathrm{KW}]$ $=12.13, \mathrm{p}=0.002$; Table 1). The age of onset in longstay inpatients $(21.3 \pm 6.2$ years $)$ was significantly lower than that of the outpatients $(30.2 \pm 11.7$ years) (Bonferroni correction test, $p=0.002$; Table 1$)$. There was also a significant difference observed in the percentage of married patients between the long-stay inpatients and the outpatients (Fisher's exact test, $\mathrm{p}=0.016$; Table 1). A significant difference was observed in the AQ subscale "attention to detail" scores among the three groups (KW test $=6.02, \mathrm{p}=0.49$; Supplementary Table 1). The shortstay inpatients obtained higher scores on the "attention to detail" subscale than the outpatients (non-corrected $\mathrm{p}=$ 0.02), but after a post hoc analysis, the p-value did not reach the significance level (Bonferroni correction test, $p=$ 0.61 ). Therefore, finally, there were no differences in the scores between the three groups.

\section{Differences in Autistic Symptoms and Self-Stigma Based on Patient Demographics}

There was a significantly negative correlation between the AQ total scores and age of onset $(r=-0.298, p<0.05$; Supplementary Table 2). Female patients with schizophrenia spectrum disorders showed a higher ISMI total score than males (unpaired $t$-test, $\mathrm{t}=-2.122, \mathrm{p}=0.036$; Table 2). Further, on the "alienation" and "social withdrawal," subscales, female patients had higher scores than males $(\mathrm{t}=$ $-2.13, \mathrm{p}=0.035$ and $\mathrm{t}=-2.483, \mathrm{p}=0.014$, respectively; Table 2). The total AQ scores and the "communication skills" and "imagination" subscale scores were significantly different among the three groups depending on the marital status of the participants (KW test, $\mathrm{KW}=10.052$, $\mathrm{p}=0.018 ; \mathrm{KW}=14.276, \mathrm{p}=0.003 ;$ and $\mathrm{KW}=8.314, \mathrm{p}=$ 0.04, respectively; Table 2). Single/unmarried patients with schizophrenia spectrum disorders showed a higher AQ total score, and higher "communication skills" and "imagination" subscale scores than married patients (Bonferroni correction test, $\mathrm{p}=0.022, \mathrm{p}=0.007$, and $\mathrm{p}=0.039$, respectively).

\section{Association Between Autistic Symptoms and Self-Stigma}

Spearman correlation analysis showed a significant positive correlation between the ISMI total scores and the AQ total scores, as well as two subscale scores, "attention switching" and "communication skills" $(\mathrm{r}=0.432$, $\mathrm{p}<0.01 ; \mathrm{r}=0.358 ; \mathrm{p}<0.01 ;$ and $\mathrm{r}=0.33, \mathrm{p}<0.01$, respectively; Table 3). A significant positive association was also observed between the AQ total score and four subscale scores of ISMI (all but "stigma resistance"), as shown in Table 3. There was also a positive correlation between the AQ subscale of "attention switching" and the ISMI subscale of "alienation" $(\mathrm{r}=0.337, \mathrm{p}<0.01)$. Further, a positive correlation was found between the AQ "communication skills" subscale and the ISMI "perceived discrimination" subscale $(\mathrm{r}=0.392, \mathrm{p}<0.01$; Table 3$)$. A multiple regression analysis controlling for age and gender revealed that the total score of the ISMI scale was positively correlated with the AQ total score 
Table I Patient Demographics

\begin{tabular}{|c|c|c|c|c|c|}
\hline & $\begin{array}{l}\text { Total Samples } \\
(\mathrm{N}=\mid 27)\end{array}$ & $\begin{array}{l}\text { Outpatients } \\
(\mathrm{N}=55)\end{array}$ & $\begin{array}{l}\text { Short-Stay Inpatients } \\
(\mathbf{N}=50)\end{array}$ & $\begin{array}{l}\text { Long-Stay Inpatients } \\
(\mathrm{N}=\mathbf{2 2})\end{array}$ & p value \\
\hline Gender (male/female) ${ }^{\mathrm{a}}$ & $63 / 64$ & $22 / 33$ & $26 / 24$ & $15 / 7$ & 0.08 \\
\hline $\mathrm{Age}^{\mathrm{b}}$ & $46.48 \pm 13.79$ & $47.80 \pm 14.22$ & $46.10 \pm 12.37$ & $44.04 \pm|5.9|$ & 0.55 \\
\hline Diagnosis $^{\mathrm{a}}$ & & & & & 0.76 \\
\hline Schizophrenia & 124 & 48 & 54 & 21 & \\
\hline Schizoaffective disorder & 3 & 1 & 1 & 1 & \\
\hline Delusional disorder & 1 & I & 0 & 0 & \\
\hline Age of onset ${ }^{c}$ & $27.48 \pm 11.08$ & $30.16 \pm 11.73$ & $27.28 \pm 11.05$ & $21.3 \pm 6.2$ & $<0.01$ \\
\hline $\begin{array}{l}\text { Duration of hospitalization } \\
\text { (days) }\end{array}$ & $629.94 \pm 1666.37$ & & $53 \pm 66.0$ & $1931.36 \pm 2609.82$ & \\
\hline Number of hospitalizations ${ }^{c}$ & $4.03 \pm 4.83$ & $3.45 \pm 4.66$ & $4.66 \pm 5.52$ & $4.16 \pm 3.27$ & 0.32 \\
\hline Education years ${ }^{\mathrm{a}}$ & & & & & 0.18 \\
\hline$\leqq 9$ & 40 & 15 & 17 & 8 & \\
\hline$>9$ to $\leqq 12$ & 51 & 23 & 16 & 12 & \\
\hline$>12$ & 35 & 17 & 16 & 2 & \\
\hline Marital status ${ }^{\mathrm{a}}$ & & & & & 0.02 \\
\hline Single & 93 & 34 & 37 & 22 & \\
\hline Married & 20 & 13 & 7 & 0 & \\
\hline Divorced & 13 & 7 & 6 & 0 & \\
\hline Widow & 1 & I & 0 & 0 & \\
\hline Partners ${ }^{\mathrm{a}}$ & & & & & 0.91 \\
\hline Yes & 102 & 45 & 39 & 18 & \\
\hline No & 25 & 10 & II & 4 & \\
\hline
\end{tabular}

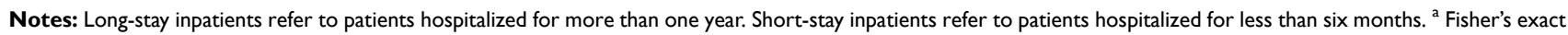
test $p$-values for gender, diagnosis, years of education, marital status, and partners among the three groups. ${ }^{b}$ Analysis of variance $p$-values for the difference in age between the three groups. ${ }^{C}$ Kruskal-Wallis test $\mathrm{p}$-values for the number of hospitalizations among the three groups.

$(\beta=0.419, \mathrm{p}<0.001 ;$ Table 4$)$, as well as gender $(\beta=0.19$, $\mathrm{p}=0.02$; Table 4).

\section{Discussion}

We hypothesized that schizophrenia spectrum disorder patients with a higher level of autistic symptoms will have difficulty coping with stigmatization due to lower communication skills and reduced cognitive flexibility, and will be more likely to internalize stigma than those with fewer autistic symptoms. To verify our hypothesis, we investigated the association between autistic symptoms and self-stigma in patients with schizophrenia spectrum disorders.

Consistent with our hypothesis, the results indicated a positive correlation between the total scores of AQ and ISMI in patients with schizophrenia spectrum disorders. The current study highlights the importance of considering autistic symptoms in the assessment and management of selfstigma in patients with schizophrenia spectrum disorders.
This study also found gender differences in self-stigma (ISMI total scores and "alienation" and "social withdrawal" subscale scores). Female patients showed a higher level of stigmatization than male patients. This result is consistent with the findings of previous studies. ${ }^{15,16}$ Gender differences in self-stigma may be partly explained by the fact that women may fear more of not being preferred as spouses due to the social stigma associated with the disorders. ${ }^{16}$ However, another study did not observe any gender differences in self-stigma. ${ }^{27}$ The discrepancy in these results may be due to cultural differences across various regions where the studies were conducted. ${ }^{27}$

In the present study, the total score on the AQ and the scores of the "communication skills" and "imagination" subscales were found to be higher in single/unmarried patients than in married patients. A recent study has reported that there were significantly more single individuals among patients with autistic spectrum disorders than among patients with schizophrenia spectrum disorders. ${ }^{28}$ 
Table 2 Comparison of Self-Stigma and Autistic Symptoms According to Patient Characteristics

\begin{tabular}{|c|c|c|c|c|c|c|}
\hline & $\begin{array}{l}\text { ISMI Total } \\
\text { Score }\end{array}$ & Alienation & $\begin{array}{l}\text { Stereotype } \\
\text { Endorsement }\end{array}$ & $\begin{array}{l}\text { Perceived } \\
\text { Discrimination }\end{array}$ & $\begin{array}{l}\text { Social } \\
\text { Withdrawal }\end{array}$ & $\begin{array}{l}\text { Stigma } \\
\text { Resistance }\end{array}$ \\
\hline \multicolumn{7}{|l|}{ Gender } \\
\hline Male & $2.16 \pm 0.46$ & $2.16 \pm 0.69$ & $2.00 \pm 0.53$ & $2.03 \pm 0.57$ & $2.06 \pm 0.63$ & $2.63 \pm 0.52$ \\
\hline Female & $2.34 \pm 0.51$ & $2.43 \pm 0.75$ & $2.10 \pm 0.62$ & $2.21 \pm 0.62$ & $2.36 \pm 0.74$ & $2.73 \pm 0.53$ \\
\hline Statistical analysis & $\begin{array}{l}\text { Unpaired } \\
t \text {-test } \\
\mathrm{t}=-2.12 \\
\mathrm{p}=0.04\end{array}$ & $\begin{array}{l}\text { Unpaired } \\
t \text {-test } \\
\mathrm{t}=-2.13 \\
\mathrm{P}=0.04\end{array}$ & $\begin{array}{l}\text { Unpaired } \\
t \text {-test } \\
\mathrm{t}=-0.89 \\
\mathrm{p}=0.38\end{array}$ & $\begin{array}{l}\text { Unpaired } \\
t \text {-test } \\
\mathrm{t}=-1.68 \\
\mathrm{p}=0.10\end{array}$ & $\begin{array}{l}\text { Unpaired } \\
t \text {-test } \\
\mathrm{t}=-2.48 \\
\mathrm{P}=0.01\end{array}$ & $\begin{array}{l}\text { Unpaired } \\
t \text {-test } \\
\mathrm{t}=-1.04 \\
\mathrm{P}=0.30\end{array}$ \\
\hline \multicolumn{7}{|l|}{ Education years } \\
\hline$\leqq 9$ & $2.26 \pm 0.46$ & $2.28 \pm 0.69$ & $2.06 \pm 0.57$ & $2.10 \pm 0.60$ & $2.26 \pm 0.69$ & $2.70 \pm 0.62$ \\
\hline $9<$ to $\leqq 12$ & $2.19 \pm 0.43$ & $2.21 \pm 0.66$ & $2.02 \pm 0.533$ & $2.13 \pm 0.53$ & $2.09 \pm 0.61$ & $2.6 I \pm 0.44$ \\
\hline$>12$ & $2.36 \pm 0.58$ & $2.46 \pm 0.85$ & $2.11 \pm 0.64$ & $2.18 \pm 0.69$ & $2.78 \pm 0.51$ & $2.78 \pm 0.51$ \\
\hline Statistical analysis & $\begin{array}{l}\mathrm{KW} \text { test } \\
\mathrm{KW}=2.24 \mathrm{p}=0.33\end{array}$ & $\begin{array}{l}\mathrm{KW} \text { test } \\
\mathrm{KW}=1.79 \mathrm{p}=0.41\end{array}$ & $\begin{array}{l}\mathrm{KW} \text { test } \\
\mathrm{KW}=0.56 \\
\mathrm{P}=0.76\end{array}$ & $\begin{array}{l}\mathrm{KW} \text { test } \\
\mathrm{KW}=0.43 \\
\mathrm{P}=0.81\end{array}$ & $\begin{array}{l}\mathrm{KW} \text { test } \\
\mathrm{KW}=2.65 \\
\mathrm{P}=0.27\end{array}$ & $\begin{array}{l}\mathrm{KW} \text { test } \\
\mathrm{KW}=3.92 \\
\mathrm{P}=0.14\end{array}$ \\
\hline \multicolumn{7}{|l|}{ Marital status } \\
\hline Single & $2.25 \pm 0.49$ & $2,29 \pm 0.75$ & $2.08 \pm 0.59$ & $2.13 \pm 0.61$ & $2.20 \pm 0.69$ & $2.65 \pm 0.57$ \\
\hline Married & $2.21 \pm 0.43$ & $2.26 \pm 0.62$ & $1.93 \pm 0.46$ & $2.07 \pm 0.52$ & $2.21 \pm 0.60$ & $2.69 \pm 0.33$ \\
\hline Divorced & $2.31 \pm 0.65$ & $2.33 \pm 0.86$ & $2.00 \pm 0.69$ & $2.18 \pm 0.70$ & $2.30 \pm 0.95$ & $2.85 \pm 0.38$ \\
\hline Widow & 2.31 & 2.5 & 2 & 1.6 & 2.4 & 3.2 \\
\hline Statistical analysis & $\begin{array}{l}\mathrm{KW} \text { test } \\
\mathrm{KW}=0.28 \mathrm{p}=0.96\end{array}$ & $\begin{array}{l}\mathrm{KW} \text { test } \\
\mathrm{KW}=0.30 \\
\mathrm{P}=0.96\end{array}$ & $\begin{array}{l}\mathrm{KW} \text { test } \\
\mathrm{KW}=1.15 \\
\mathrm{P}=0.766\end{array}$ & $\begin{array}{l}\mathrm{KW} \text { test } \\
\mathrm{KW}=2.15 \\
\mathrm{P}=0.54\end{array}$ & $\begin{array}{l}\mathrm{KW} \text { test } \\
\mathrm{KW}=0.29 \\
\mathrm{P}=0.96\end{array}$ & $\begin{array}{l}\mathrm{KW} \text { test } \\
\mathrm{KW}=3.67 \\
\mathrm{P}=0.30\end{array}$ \\
\hline \multicolumn{7}{|l|}{ Partners } \\
\hline Yes & $2.27 \pm 0.50$ & $2.32 \pm 0.75$ & $2.07 \pm 0.59$ & $2.13 \pm 0.60$ & $2.23 \pm 0.71$ & $2.70 \pm 0.53$ \\
\hline No & $2.18 \pm 0.49$ & $2.19 \pm 0.67$ & $1.97 \pm 0.52$ & $2.08 \pm 0.61$ & $2.15 \pm 0.66$ & $2.62 \pm 0.49$ \\
\hline \multirow[t]{2}{*}{ Statistical analysis } & $\begin{array}{l}M W \text { test } \\
M W=1115.50 \\
P=0.333\end{array}$ & $\begin{array}{l}M W \text { test } \\
M W=1100.50 \\
P=0.288\end{array}$ & $\begin{array}{l}M W \text { test } \\
M W=1174.00 \\
P=0.538\end{array}$ & $\begin{array}{l}M W \text { test } \\
M W=1211.00, \\
P=0.696\end{array}$ & $\begin{array}{l}M W \text { test } \\
M W=1190.00, \\
P=0.605\end{array}$ & $\begin{array}{l}M W \text { test } \\
M W=1119.50, \\
p=0.340\end{array}$ \\
\hline & AQ total score & Social skills & $\begin{array}{l}\text { Attention } \\
\text { switching }\end{array}$ & Attention to detail & $\begin{array}{l}\text { communication } \\
\text { skills }\end{array}$ & Imagination \\
\hline \multicolumn{7}{|l|}{ Gender } \\
\hline Male & $23.95 \pm 5.78$ & $4.54 \pm 2.62$ & $5.25 \pm 1.69$ & $5.19 \pm 1.85$ & $4.46 \pm 2.04$ & $4.63 \pm 1.82$ \\
\hline Female & $24.18 \pm 6.86$ & $5.11 \pm 2.16$ & $5.25 \pm 1.93$ & $5.22 \pm 2.37$ & $4.08 \pm 2.56$ & $4.4 I \pm 2.16$ \\
\hline Statistical analysis & $\begin{array}{l}\text { Unpaired } \\
\text { test } \\
t=0.02 p=0.99\end{array}$ & $\begin{array}{l}\text { Unpaired } \\
\text { test } \\
\mathrm{t}=-1.33 \\
\mathrm{p}=0.19\end{array}$ & $\begin{array}{l}\text { Unpaired } \\
\text { test } \\
\mathrm{t}=0.01 \\
\mathrm{p}=0.99\end{array}$ & $\begin{array}{l}\text { Unpaired } \\
\text { test } \\
\mathrm{t}=-0.08 \\
\mathrm{p}=0.94\end{array}$ & $\begin{array}{l}\text { Unpaired } \\
\text { test } \\
\mathrm{t}=0.93 \\
\mathrm{p}=0.35\end{array}$ & $\begin{array}{l}\text { Unpaired } \\
\text { test } \\
\mathrm{t}=0.64 \\
\mathrm{p}=0.52\end{array}$ \\
\hline \multicolumn{7}{|l|}{ Education years } \\
\hline$\leqq 9$ & $24.28 \pm 6.5 \mathrm{I}$ & $5.20 \pm 2.42$ & $5.05 \pm 1.63$ & $4.90 \pm 2.11$ & $4.33 \pm 2.29$ & $4.80 \pm 1.92$ \\
\hline $9<\leqq 12$ & $24.29 \pm 6.49$ & $4.90 \pm 2.44$ & $5.47 \pm 1.99$ & $5.43 \pm 1.90$ & $4.45 \pm 2.37$ & $4.04 \pm 2.02$ \\
\hline$>12$ & $23.77 \pm 5.92$ & $4.43 \pm 2.27$ & $5.20 \pm 1.75$ & $5.14 \pm 2.41$ & $4.03 \pm 2.27$ & $4.97 \pm 1.93$ \\
\hline Statistical analysis & $\begin{array}{l}\mathrm{KW} \text { test } \\
\mathrm{KW}=0.04 \mathrm{p}=0.98\end{array}$ & $\begin{array}{l}\mathrm{KW} \text { test } \\
\mathrm{KW}=1.40 \\
\mathrm{P}=0.50\end{array}$ & $\begin{array}{l}\mathrm{KW} \text { test } \\
\mathrm{KW}=0.42 \mathrm{p}=0.80\end{array}$ & $\begin{array}{l}\mathrm{KW} \text { test } \\
\mathrm{KW}=\mathrm{I} .5 \mathrm{I} \\
\mathrm{P}=0.47\end{array}$ & $\begin{array}{l}\mathrm{KW} \text { test } \\
\mathrm{KW}=0.37 \\
\mathrm{P}=0.83\end{array}$ & $\begin{array}{l}K W \text { test } \\
K W=5.16 \\
P=0.08\end{array}$ \\
\hline $\begin{array}{l}\text { Marital status } \\
\text { Single }\end{array}$ & $25.11 \pm 6.13$ & $4.91 \pm 2.45$ & $5.38 \pm 1.73$ & $5.31 \pm 2.12$ & $4.70 \pm 2.16$ & $4.80 \pm 1.85$ \\
\hline
\end{tabular}

(Continued) 
Table 2 (Continued).

\begin{tabular}{|c|c|c|c|c|c|c|}
\hline & $\begin{array}{l}\text { ISMI Total } \\
\text { Score }\end{array}$ & Alienation & $\begin{array}{l}\text { Stereotype } \\
\text { Endorsement }\end{array}$ & $\begin{array}{l}\text { Perceived } \\
\text { Discrimination }\end{array}$ & $\begin{array}{l}\text { Social } \\
\text { Withdrawal }\end{array}$ & $\begin{array}{l}\text { Stigma } \\
\text { Resistance }\end{array}$ \\
\hline $\begin{array}{l}\text { Married } \\
\text { Divorced } \\
\text { Widow }\end{array}$ & $\begin{array}{l}20.45 \pm 6.72 \\
22.54 \pm 5.22 \\
20\end{array}$ & $\begin{array}{l}4.55 \pm 2.42 \\
4.69 \pm 2.39 \\
4\end{array}$ & $\begin{array}{l}4.80 \pm 1.85 \\
4.85 \pm 2.12 \\
8\end{array}$ & $\begin{array}{l}4.80 \pm 1.79 \\
5.15 \pm 2.67 \\
4\end{array}$ & $\begin{array}{l}2.90 \pm 2.65 \\
3.54 \pm 1.85 \\
\text { । }\end{array}$ & $\begin{array}{l}3.4 \pm 2.04 \\
4.31 \pm 2.50 \\
3\end{array}$ \\
\hline Statistical analysis & $\begin{array}{l}\mathrm{KW} \text { test } \\
\mathrm{KW}=10.05 \\
\mathrm{P}=0.02\end{array}$ & $\begin{array}{l}\mathrm{KW} \text { test } \\
\mathrm{KW}=0.48 \\
\mathrm{P}=0.92\end{array}$ & $\begin{array}{l}\mathrm{KW} \text { test } \\
\mathrm{KW}=5.02 \\
\mathrm{P}=0.17\end{array}$ & $\begin{array}{l}\mathrm{KW} \text { test } \\
\mathrm{KW}=1.35 \\
\mathrm{P}=0.72\end{array}$ & $\begin{array}{l}\mathrm{KW} \text { test } \\
\mathrm{KW}=14.28 \\
\mathrm{P}=0.003\end{array}$ & $\begin{array}{l}\mathrm{KW} \text { test } \\
\mathrm{KW}=8.31 \\
\mathrm{p}=0.04\end{array}$ \\
\hline $\begin{array}{l}\text { Partners } \\
\text { Yes } \\
\text { No }\end{array}$ & $\begin{array}{l}23.79 \pm 6.49 \\
25.2 \pm 5.58\end{array}$ & $\begin{array}{l}4.74 \pm 2.43 \\
5.20 \pm 2.36\end{array}$ & $\begin{array}{l}5.22 \pm 2.13 \\
5.16 \pm 2.11\end{array}$ & $\begin{array}{l}5.22 \pm 2.13 \\
5.16 \pm 2.11\end{array}$ & $\begin{array}{l}4.22 \pm 2.35 \\
4.48 \pm 2.18\end{array}$ & $\begin{array}{l}4.41 \pm 2.02 \\
4.96 \pm 1.90\end{array}$ \\
\hline Statistical analysis & $\begin{array}{l}M W \text { test } \\
M W=1456.50 \\
P=0.27\end{array}$ & $\begin{array}{l}M W \text { test } \\
M W=|4| 0.50 \\
P=0.4 \mid\end{array}$ & $\begin{array}{l}M W \text { test } \\
M W=1362.00 \\
p=0.59\end{array}$ & $\begin{array}{l}M W \text { test } \\
M W=1255.50 \\
p=0.905\end{array}$ & $\begin{array}{l}M W \text { test } \\
M W=1384.50 \\
p=0.503\end{array}$ & $\begin{array}{l}M W \text { test } \\
M W=|49| .50 \\
P=0.184\end{array}$ \\
\hline
\end{tabular}

Notes: The table show the p-values for the unpaired $t$-test, Kruskal-Wallis test, and Mann-Whitney U-test to compare self-stigma and autistic symptoms according to patient characteristics.

Abbreviations: IMSI, Internalized Stigma of Mental Illness; AQ, Autism-Spectrum Quotient; KW, Kruskal-Wallis test; MW, Mann-Whitney U-test.

Table 3 Bivariate Correlations Between Self-Stigma and Autistic Symptoms

\begin{tabular}{|c|l|l|l|l|l|l|}
\hline & $\begin{array}{l}\text { ISMI Total } \\
\text { Score }\end{array}$ & Alienation & $\begin{array}{l}\text { Stereotype } \\
\text { Endorsement }\end{array}$ & $\begin{array}{l}\text { Perceived } \\
\text { Discrimination }\end{array}$ & $\begin{array}{l}\text { Social } \\
\text { Withdrawal }\end{array}$ & $\begin{array}{l}\text { Stigma } \\
\text { Resistance }\end{array}$ \\
\hline AQ total score & $\mathbf{0 . 4 3 * *}$ & $\mathbf{0 . 3 2 *}$ & $\mathbf{0 . 4 0 * *}$ & $\mathbf{0 . 4 2 * *}$ & $\mathbf{0 . 3 8 * *}$ & 0.04 \\
Social skills & 0.28 & 0.14 & 0.22 & 0.24 & 0.25 & 0.20 \\
Attention switching & $\mathbf{0 . 3 6 * *}$ & $\mathbf{0 . 3 4 * *}$ & $\mathbf{0 . 3 2 *}$ & 0.28 & $\mathbf{0 . 3 2} *$ & 0.03 \\
Attention to detail & 0.06 & 0.11 & 0.05 & 0.08 & 0.07 & -0.10 \\
Communication skills & $\mathbf{0 . 3 3} * *$ & 0.22 & 0.29 & $\mathbf{0 . 3 9 * *}$ & 0.28 & -0.01 \\
Imagination & 0.26 & 0.18 & 0.30 & 0.26 & 0.20 & 0.02 \\
\hline
\end{tabular}

Notes: The data shows Spearman's rank correlation coefficients. Bold values represent statistical significance at the $p<0.05$ level. ${ }^{*} p<0.05, * * p<0.01$ Abbreviations: IMSI, Internalized Stigma of Mental IIIness scale; AQ, Autism-Spectrum Quotient.

Therefore, the results suggest patients with a higher level of autistic symptoms more often tend to be single.

There was a significant negative correlation between the AQ total score and age of onset in the current study. Barlati et al reported a lower age of onset in schizophrenia spectrum patients with autism spectrum disorders than those without them. ${ }^{29}$ The results of this study showed that a younger age of onset among patients with schizophrenia spectrum disorders was associated with a higher AQ-J score, which is consistent with the previous study reported by Barlati et al.

The results of this study showed a significant positive association between the total scores of the AQ and ISMI in patients with schizophrenia spectrum disorders. Furthermore, the results of the Spearman bivariate correlation analysis between the subscale scores of AQ and total score of ISMI, a significant correlation was observed between the total scores of ISMI and the AQ "attention switching" and "communication skills subscale scores. These findings suggest that difficulties in switching attention and communication skill problems, both of which are significant autistic traits, may be primarily involved in the internalization of stigma in patients with schizophrenia spectrum disorders with autistic symptoms. The results of this study suggest that patients with difficulty switching attention and communicating with others are more likely to internalize stigma due to their inability to cope with the social stigma. However, to our knowledge there seems to be no prior study that supports our findings. In the future, it may be worth conducting a cohort research study on coping with stigma in patients who have inherent difficulty in switching attention and communication problems.

\section{Limitations of the Study}

There were several limitations of the present study. First, it was not possible to describe a causal relationship between 
Table 4 Multiple Regression Analysis Assessing the Association Between ISMI, AQ, Age, and Gender

\begin{tabular}{|c|c|c|c|c|c|}
\hline & \multicolumn{2}{|c|}{ ISMI-J Total Score } & \multirow[t]{2}{*}{$\beta$} & \multirow[t]{2}{*}{$\mathbf{t}$} & \multirow[t]{2}{*}{ p value } \\
\hline & B & SE & & & \\
\hline Age & $<0.001$ & 0.003 & -0.004 & -0.05 & 0.96 \\
\hline Gender & 0.18 & 0.08 & 0.19 & 2.31 & 0.02 \\
\hline AQ total score & 0.03 & $<0.01$ & 0.42 & 5.18 & $<0.001$ \\
\hline \multirow[t]{2}{*}{ Model summary } & $R$ & $R^{2}$ & Adjusted $R^{2}$ & \multicolumn{2}{|c|}{ Std. Error of the Estimates } \\
\hline & $\begin{array}{l}0.46 \\
R^{2} \text { change } \\
0.21\end{array}$ & $\begin{array}{l}0.21 \\
F \text { change } \\
10.96\end{array}$ & $\begin{array}{l}0.19 \\
\text { Significance. } F \text { change } \\
<0.001\end{array}$ & 0.45 & \\
\hline
\end{tabular}

Note: Bold values represent statistical significance at the $p<0.05$ level.

Abbreviations: IMSI, Internalized Stigma of Mental Illness scale; AQ, Autism-Spectrum Quotient; SE, standard error; $R$, multiple correlation coefficient; $B$, partial regression coefficient.

autistic symptoms and self-stigma because of the crosssectional nature of the study. Second, the results cannot be generalized to the entire target population because the data were obtained from a single medical institution. Third, since the current study used self-report rating scales, the scores might have been affected by patient fatigue, mental status, and participants' motivation at the time of assessment. However, the patients' mental state has not been evaluated using the Brief Psychiatric Rating Scale or Positive and Negative Syndrome Scale. Moreover, we did not evaluate two possible confounders: depression and insight. Depressive symptoms can strongly overlap with features of $\mathrm{AQ}$ (patients may not be rating their actual autistic traits, but rather their social withdrawal and ruminative nature). Self-stigma has been found to be associated with insight in schizophrenia, as well as with depression, ${ }^{30}$ In future, multiple regression analyses between autistic symptoms and self-stigma using depressive symptoms and insight as confounding factors will be required. Potential cognitive impairment in patients may also reduce the validity of responses on self-report measures. Finally, we performed an association analysis of schizophrenia spectrum disorders, which included delusional disorders, although it may not be necessarily have been the best choice.

\section{Conclusion}

Self-stigma may lead to a negative influence on QoL, selfefficacy, treatment adherence, and recovery in patients with schizophrenia spectrum disorders. Therefore, it is worth investigating the factors that influence patients' selfstigma, in order to assess and manage self-stigma among these patients.
This is the first study to reveal the positive association between autistic symptoms and self-stigma in schizophrenia spectrum disorders.

The findings suggest that a higher level of self-stigma may exist in patients with schizophrenia spectrum disorders who have autistic symptoms (especially the problem of attention switching and communication skills) as they may serve as an inhibitor of recovery and self-efficacy.

Our study's findings direct the attention of the clinicians towards the importance of assessing and managing self-stigma, especially in schizophrenic patients with autism spectrum disorders.

\section{Data Sharing Statement}

The data that support the results reported in the current study are available on request from the corresponding author.

\section{Acknowledgments}

The authors are very thankful to the patients and their family. We would also like to thank Editage for English language editing.

\section{Funding}

This study did not receive any external funding.

\section{Disclosure}

Hiroshi Komatsu reports personal fees from Dainippon Sumitomo Pharma Co., Ltd., Meiji Seika Pharma Co., Ltd., Otsuka Pharmaceutical Co., Ltd., Janssen Pharmaceutical K. K., and Yoshitomi Pharmaceutical Industries, Ltd., outside the submitted work.

Takashi Ono reports personal fees from Dainippon Sumitomo Pharma Co., Ltd.,Otsuka Pharmaceutical Co., 
Ltd., Janssen Pharmaceutical K.K., and Yoshitomi Pharmaceutical Industries, Ltd., outside the submitted work.

Yusuke Ishida reports personal fees from Otsuka Pharmaceutical Co., Ltd., Sumitomo Dainippon Pharma Co., Ltd., Eisai Co., Ltd., and Janssen Pharmaceutical K.K., outside the submitted work. Yoshihisa Kakuto reports personal fees from Otsuka Pharmaceutical Co., Ltd,Janssen Pharmaceutical K.K., and Sumitomo Dainippon Pharma Co., Ltd., outside the submitted work.

The authors report no other potential conflicts of interest for this work.

\section{References}

1. Thornicroft G, Brohan E, Rose D, Sartorius N, Leese M, INDIGO Study Group. Global pattern of experienced and anticipated discrimination against people with schizophrenia: a cross-sectional survey. Lancet. 2009;373(9661):408-415. doi:10.1016/S0140-6736(08) 61817-6

2. Dickerson FB, Sommerville J, Origoni AE, Ringel NB, Parente F. Experiences of stigma among outpatients with schizophrenia. Schizophr Bull. 2002;28(1):143-155. doi:10.1093/oxfordjournals. schbul.a006917

3. Lee S, Chiu MY, Tsang A, Chui H, Kleinman A. Stigmatizing experience and structural discrimination associated with the treatment of schizophrenia in Hong Kong. Soc Sci Med. 2006;62 (7):1685-1696. doi:10.1016/j.socscimed.2005.08.016

4. Corrigan PW, Wassel A. Understanding and influencing the stigma of mental illness. J Psychosoc Nurs Ment Health Serv. 2008;46 (1):42-48. doi:10.3928/02793695-20080101-04

5. Corrigan PW, Watson AC. Understanding the impact of stigma on people with mental illness. World Psychiatry. 2002;1(1):16-20.

6. Watson AC, Corrigan P, Larson JE, Sells M. Self-stigma in people with mental illness. Schizophr Bull. 2007;33(6):1312-1318.

7. Livingston JD, Boyd JE. Correlates and consequences of internalized stigma for people living with mental illness: a systematic review and meta-analysis. Soc Sci Med. 2010;71(12):2150-2161. doi:10.1016/j. socscimed.2010.09.030

8. Karakaş SA, Okanlı A, Yılmaz E. The effect of internalized stigma on the self esteem in patients with schizophrenia. Arch Psychiatr Nurs. 2016;30(6):648-652. doi:10.1016/j.apnu.2016.02.006

9. Yanos PT, Roe D, Markus K, Lysaker PH. Pathways between internalized stigma and outcomes related to recovery in schizophrenia spectrum disorders. Psychiatr Serv. 2008;59(12):1437-1442. doi:10.1176/ps.2008.59.12.1437

10. Vrbova K, Prasko J, Ociskova M, et al. Quality of life, self-stigma, and hope in schizophrenia spectrum disorders: a cross-sectional study. Neuropsychiatr Dis Treat. 2017;13:567-576.

11. Ritsher JB, Otilingam PG, Grajales M. Internalized stigma of mental illness: psychometric properties of a new measure. Psychiatry Res. 2003;121(1):31-49.

12. Morgades-Bamba CI, Fuster-Ruizdeapodaca MJ, Molero F. Internalized stigma and its impact on schizophrenia quality of life. Psychol Health Med. 2019;24(8):992-1004. doi:10.1080/ 13548506.2019.1612076

13. Oexle N, Muller M, Kawohl W, et al. Self-stigma as a barrier to recovery: a longitudinal study. Eur Arch Psychiatry Clin Neurosci. 2018;268(2):209-212. doi:10.1007/s00406-017-0773-2
14. Fung KMT, Tsang HWH, Corrigan PW. Self-stigma of people with schizophrenia as predictor of their adherence to psychosocial treatment. Psychiatr Rehabil J. 2008;32(2):95-104. doi:10.2975/ 32.2.2008.95.104

15. Yilmaz E, Okanli A. The effect of internalized stigma on the adherence to treatment in patients with schizophrenia. Arch Psychiatr Nurs. 2015;29(5):297-301. doi:10.1016/j.apnu.2015.05.006

16. Çapar M, Kavak F. Effect of internalized stigma on functional recovery in patients with schizophrenia. Perspect Psychiatr Care. 2019;55 (1):103-111. doi:10.1111/ppc.12309

17. Kamaradova D, Latalova K, Prasko J, et al. Connection between self-stigma, adherence to treatment, and discontinuation of medication. Patient Prefer Adherence. 2016;10:1289-1298. doi:10.2147/PPA.S99136

18. Camp DL, Finlay WM, Lyons E. Is low self-esteem an inevitable consequence of stigma? An example from women with chronic mental health problems. Soc Sci Med. 2002;55(5):823-834. doi:10.1016/S0277-9536(01)00205-2

19. Vrbova K, Prasko J, Ociskova M, et al. Suicidality, self-stigma, social anxiety and personality traits in stabilized schizophrenia patients - a cross-sectional study. Neuropsychiatr Dis Treat. 2018;14:1415-1424. doi:10.2147/NDT.S162070

20. Vrbova K, Prasko J, Holubova M, Slepecky M, Ociskova M. Positive and negative symptoms in schizophrenia and their relation to depression, anxiety, hope, self-stigma and personality traits - a cross-sectional study. Neuro Endocrinol Lett. 2018;39(1):9-18.

21. Margetić BA, Jakovljević M, Ivanec D, Margetić B, Tošić G. Relations of internalized stigma with temperament and character in patients with schizophrenia. Compr Psychiatry. 2010;51(6):603-606. doi:10.1016/j.comppsych.2010.02.010

22. Murai T, Matsukawa N, Sasamoto A. Pathology of social brain and social cognitive impairments in schizophrenia. Seishin Shinkeigaku Zasshi. 2012;114(8):915-920.

23. De Crescenzo F, Postorino V, Siracusano M, et al. Autistic symptoms in schizophrenia spectrum disorders: a systematic review and meta-analysis. Front Psychiatry. 2019;10:78. doi:10.3389/ fpsyt.2019.00078

24. Tanabe Y, Hayashi K, Ideno Y. The Internalized Stigma of Mental Illness (ISMI) scale: validation of the Japanese version. $B M C$ Psychiatry. 2016;16:116. doi:10.1186/s12888-016-0825-6

25. Baron-Cohen S, Wheelwright S, Skinner R, Martin J, Clubley E. The autism-spectrum quotient (AQ): evidence from Asperger syndrome/ high-functioning autism, males and females, scientists and mathematicians. J Autism Dev Disord. 2001;31(1):5-17.

26. Wakabayashi A, Tojo Y, Baron-Cohen S, Wheelwright S. The autism-spectrum quotient (AQ) Japanese version: evidence from high-functioning clinical group and normal adults. Shinrigaku Kenkyu. 2004;75(1):78-84. doi:10.4992/jjpsy.75.78

27. Vrbova K, Prasko J, Holubova M, et al. Self-stigma and schizophrenia: a cross-sectional study. Neuropsychiatr Dis Treat. 2016;12:3011-3020. doi:10.2147/NDT.S120298

28. Fusar-Poli L, Ciancio A, Gabbiadini A, et al. Self-reported autistic traits using the AQ: A comparison between individuals with asd, psychosis, and non-clinical controls. Brain Sci. 2020;10(5):291. doi: 10.3390/brainsci10050291

29. Barlati S, Deste G, Gregorelli M, Vita A. Autistic traits in a sample of adult patients with schizophrenia: prevalence and correlates. Psychol Med. 2019;49:140-148. doi:10.1017/S0033291718000600

30. Belvederi Murri M, Amore M, Calcagno P, et al. The "insight paradox" in schizophrenia: magnitude, moderators and mediators of the association between insight and depression. Schizophr Bull. 2016;42 (5):1225-1233. doi:10.1093/schbul/sbw040 


\section{Publish your work in this journal}

Neuropsychiatric Disease and Treatment is an international, peerreviewed journal of clinical therapeutics and pharmacology focusing on concise rapid reporting of clinical or pre-clinical studies on a range of neuropsychiatric and neurological disorders. This journal is indexed on PubMed Central, the 'PsycINFO' database and CAS, and is the official journal of The International Neuropsychiatric Association (INA). The manuscript management system is completely online and includes a very quick and fair peer-review system, which is all easy to use. Visit http://www.dovepress.com/testimonials.php to read real quotes from published authors.

Submit your manuscript here: https://www.dovepress.com/neuropsychiatric-disease-and-treatment-journal 